\title{
One-Pot Synthesis of Substituted Phthalazine-5,10-dione Derivatives in the Presence of Triflate Catalyst
}

\author{
Kadir TURHAN ${ }^{1 *}$
}

\begin{abstract}
In this study, substituted 1H-pyrazolo[1,2-b]phthalazine-5,10-diones which are considered that may possess biological activity have been obtained via one-pot multi-component cyclocondensation reaction of phthalhydrazide, aromatic aldehydes and malononitrile catalyzed in the presence of $\mathrm{Cu}(\mathrm{OTf})_{2}$ in very good yields and short times. The structures of all these synthesized compounds (4a-f) have been determined and characterized by infrared, nuclear magnetic resonance, mass spectral data, and elemental analysis.
\end{abstract}

Keywords: Phthalazinedione, triflate, one-pot, multicomponent, green chemistry

\footnotetext{
${ }^{1}$ Kadir TURHAN (Orcid ID: 0000-0002-7718-1618), Yıldız Teknik Üniversitesi, Fen Edebiyat Fakültesi, Kimya, İstanbul, Türkiye

*Sorumlu yazar/Corresponding Author: Kadir TURHAN, kturhan@yildiz.edu.tr 


\section{INTRODUCTION}

Nowadays, green chemistry is attracting great attention due to the global population's ability to overcome environmental pollution problems. Green chemistry, also called sustainable chemistry, is a process that reduces or eliminates the use and production of hazardous substances. From this point of view, one-pot harmful solvent-free reactions are considered as an ideal method for green synthesis (Wender et al., 1997).

Pyrazolo[1,2- $b]$ phthalazinediones are an important class of compounds that demonstrate biological activities such as analgesic, cytotoxic, cardiotonic, vasorelaxant, anti-allergic, antimicrobial, antifungal, anti-hypoxic, anticonvulsant, antipyretic, anti-inflammatory and antiviral (Wei et al., 2006; Xia et al., 2007; Vera-DiVaio et al., 2009; Lv et al., 2010; Nabid et al., 2010; Zhang et al., 2010; Raghuvanshi and Singh, 2011; Kiasat and Davarpanah, 2013; Bashti et al., 2015). Because of these properties, it is important to develop non-complex methods to obtain substituted $1 H$-pyrazolo[1,2$b]$ phthalazine-5,10-dione derivatives.

In the synthesis of substituted $1 \mathrm{H}-$ pyrazolo[1,2- $b]$ phthalazine-5,10-dione

derivatives, there are only a few studies on the synthesis of one-pot three-component phthalhydrazine, aromatic aldehydes, and malononitrile together with acidic or basic catalysts. However, most of the processes mentioned have disadvantages such as low yields, long reaction times, application of toxic or unsafe catalysts, expensive methods or more difficult reaction conditions ( $\mathrm{Lv}$ et al., 2010; Reddy and Jeong, 2013; Bashti et al., 2015; Dabholkar et al., 2017; Dabholkar et al., 2018).

Metal salts of trifluoromethanesulfonic acid (metal triflates) are a new type of Lewis acid and are powerful catalysts for several organic syntheses. Due to their high stability, water tolerance, and recoverability from water, they are frequently used in organic synthesis as a catalyst (Kobayashi et al., 2002). There are many publications on the synthesis of substituted $1 \mathrm{H}$ pyrazolo[1,2- $b]$ phthalazine-5,10-dione derivatives in the literature (Teimouri, 2006; Ghahremanzadeh et al., 2008; Torkiana et al., 2011; Shah et al., 2012; Shaterian and Mohammadnia, 2012; Song et al., 2012; Ghomi et al., 2014; Kefayati et al. 2014; Reddy et al., 2014; Vaghei et al., 2014; Vafaee et al.,2015; Kefayati et al. 2016; Roy et al., 2016; Sangani et al., 2016; Lamera et al., 2017; Piltan, 2017; Mohamadpour et al., 2018), but none of them are related to their synthesis in the presence of metal triflate catalyst. This article reports the synthesis of substituted $1 H$-pyrazolo[1,2- $b]$ phthalazine5,10 -dione derivatives catalyzed by $\mathrm{Cu}(\mathrm{OTf})_{2}$ catalyst by the one-pot multicomponent reaction starting from the corresponding phthalhydrazide with substituted benzaldehyde and malononitrile at $80{ }^{\circ} \mathrm{C}$. This synthetic method is consistent with the green chemistry approach because it defines an environmentally friendly, efficient and economical reaction that occurs at low temperatures without any harmful solvents and catalysts (Figure 1). 
<smiles>O=c1[nH][nH]c(=O)c2ccccc12</smiles>

(1)<smiles>[R]c1ccc(C=O)c([R])c1</smiles>

(2)

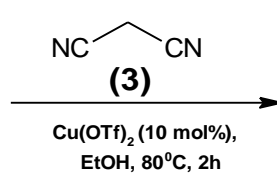

tOH, $80^{\circ} \mathrm{C}, 2 \mathrm{~h}$

$\mathrm{R}=\mathrm{H}, \mathrm{Br}, \mathrm{Cl}, \mathrm{NO}_{2}, \mathrm{OCH}_{3}, \mathrm{CH}_{3}$

$\mathrm{R}^{1}=\mathrm{H}, \mathrm{Cl}$

Figure 1. One-pot synthesis of substituted $1 H$-pyrazolo[1,2-b] phthalazine-5,10-diones

\section{MATERIALS AND METHODS}

Reagents purchased from Merck were as follows: ethanol, dichloromethane, chloroform, ethyl acetate, n-hexane, phthalhydrazide, benzaldehyde, p-bromobenzaldehyde, pnitrobenzaldehyde, p-methoxybenzaldehyde, $\mathrm{p}$ methylbenzaldehyde,

3,5-dichlorobenzaldehyde, malononitrile, copper(II) trifluoromethanesulfonic acid, silica gel 60 (0.063-0.200 mm), and sea sand. All reagents were available commercially and used without further purification in the reactions. TLC was carried out on aluminum sheets pre-coated with silica gel 60 F254 purchased from Merck, and the spots were visualized with UV light (254/366 nm) using a Camag UV lamp.

${ }^{1} \mathrm{H}$ NMR and ${ }^{13} \mathrm{C}$ NMR spectra were saved on "Bruker $500 \mathrm{MHz}$ " spectrometers, in chloroform- $d\left(\mathrm{CDCl}_{3}\right)$ or DMSO using the tetramethylsilane (TMS) standard according to the solubility of the materials. FT-IR spectra were recorded on a "Philips PU 9714 ATR spectrophotometer", using the "Perkin-Elmer Spectrum One" program. Mass spectra (MS) were obtained with $70 \mathrm{eV}$ "Hewlett Packard GC/MS 6890/5973”. Melting points were obtained with a Gallenkamp Melting Point Apparatus in open capillaries with no correction.
General Procedure for the One-Pot Synthesis of $1 H$-pyrazolo[1,2-b] phthalazine-5,10-dione Derivatives (4a-f)

A mixture of phthalhydrazide (1) (1 mmol), substituted benzaldehyde (2) (1 $\mathrm{mmol})$, malononitrile (3) $(1 \mathrm{mmol}), \mathrm{Cu}(\mathrm{OTf})_{2}(10 \mathrm{~mol}$ $\%)$ in ethanol $(5 \mathrm{~mL})$ was refluxed at $80^{\circ} \mathrm{C}$ for the appropriate time. The progress of the reaction was monitored by TLC. After completion of the reaction, the crude product was filtered and then washed with water. The solid product was purified by recrystallization procedure in ethanol or column chromatography. All the products were characterized by spectroscopic data (FTIR, ${ }^{1} \mathrm{H}$ NMR, ${ }^{13} \mathrm{C}$ NMR, MS and EA) after the purification processes.

\section{3-Amino-5,10-dioxo-1-phenyl-5,10-dihydro-} $1 H$-pyrazolo[1,2-b]phthalazine-2-carbonitrile (4a)

Yellow crystalline, mp. 272-74 ${ }^{\circ} \mathrm{C}$. FTIR(ATR): $v=3361,3313,3016,2897,2198$, 1661, 1494, 1380, $792 \mathrm{~cm}^{-1} .{ }^{1} \mathrm{H}$ NMR (DMSO$\left.d_{6}, 500 \mathrm{MHz}\right): \delta=6.13(\mathrm{~s}, 1 \mathrm{H}), 7.33(2 \mathrm{H}, \mathrm{d}$, $J=7.08 \mathrm{~Hz}$, Ar), $7.36(1 \mathrm{H}$, brd, $J=7.52 \mathrm{~Hz}, \mathrm{Ar})$, $7.46(2 \mathrm{H}, \mathrm{d}, J=6.98 \mathrm{~Hz}, \mathrm{Ar}), 7.97(2 \mathrm{H}, \mathrm{brd}$, $J=5.40 \mathrm{~Hz}, \mathrm{Ar}), 8.08\left(2 \mathrm{H}, \mathrm{s}, \mathrm{NH}_{2}\right), 8.10(1 \mathrm{H}$, brd, $J=5.70 \mathrm{~Hz}, \mathrm{Ar}), 8.27(1 \mathrm{H}$, brd, $J=5.60 \mathrm{~Hz}$, Ar) ppm. ${ }^{13} \mathrm{C}$ NMR (DMSO- $\left.d_{6}, 125 \mathrm{MHz}\right): \delta=$ 61.31, 116.03, 126.77, 127.29, 128.27, 128.52, $128.65,133.73,134.66,150.83,154.78,156.82$ ppm. GC-MS: $\mathrm{m} / \mathrm{z}=316\left[\mathrm{M}^{+}\right]$. Anal. calcd. for 
$\mathrm{C}_{18} \mathrm{H}_{12} \mathrm{~N}_{4} \mathrm{O}_{2}$ : C, 68.35; H, 3.82; N, 17.71 Found: C, 68.49; H, 4.01; N, 17.65 .

3-Amino-1-(4-bromophenyl)-5,10-dioxo-5,10dihydro-1H-pyrazolo[1,2-b] phthalazine-2carbonitrile (4b)

Light yellow crystalline, mp. 264-66 ${ }^{\circ} \mathrm{C}$. FTIR (ATR): $v=3375,3306$ 3022, 2897, 2200, 1662, 1563, 1380, 1277, $846 \mathrm{~cm}^{-1}$. ${ }^{1} \mathrm{H}$ NMR (DMSO- $\left.d_{6}, 500 \mathrm{MHz}\right): \delta=6.14(\mathrm{~s}, 1 \mathrm{H}, \mathrm{CH}), 7.46$ $(2 \mathrm{H}, \mathrm{d}, J=8.35 \mathrm{~Hz}, \mathrm{Ar}), 7.58(2 \mathrm{H}, \mathrm{d}, J=8.30 \mathrm{~Hz}$, Ar), $7.98(2 \mathrm{H}, \mathrm{dd}, J=4.65 ; 4.30 \mathrm{~Hz}, \mathrm{Ar}), 8.09$ $(1 \mathrm{H}, \mathrm{dd}, J=5.40 ; 3.55 \mathrm{~Hz}, \mathrm{Ar}), 8.14\left(2 \mathrm{H}, \mathrm{s}, \mathrm{NH}_{2}\right)$, $8.27(1 \mathrm{H}, \mathrm{dd}, J=5.05 ; 3.90 \mathrm{~Hz}, \mathrm{Ar}) \mathrm{ppm} .{ }^{13} \mathrm{C}$ NMR (DMSO- $\left.d_{6}, 125 \mathrm{MHz}\right): \delta=62.73,116.18$, $122.79,127.69,128.64,128.98,134.66,145.85$, 147.36, 150.93, 153.78, 156.71 ppm. GC-MS: $\mathrm{m} / \mathrm{z}=395\left[\mathrm{M}^{+}\right]$. Anal. calcd. for $\mathrm{C}_{18} \mathrm{H}_{11} \mathrm{BrN}_{4} \mathrm{O}_{2}$ : C, 54.70; H, 2.81; N, 14.18. Found: C, 54.98; H, $2.92 ; \mathrm{N}, 14.27$.

\section{3-Amino-1-(4-nitrophenyl)-5,10-dioxo-5,10- dihydro-1H-pyrazolo[1,2-b] phthalazine-2- carbonitrile (4c)}

Light yellow crystalline, mp. 266-68 ${ }^{\circ} \mathrm{C}$, FTIR (ATR): $v=3433,3323$ 3075, 2927, 2198, $1680,1657,1516,1380,1277,870 \mathrm{~cm}^{-1} \cdot{ }^{1} \mathrm{H}$ NMR (DMSO- $\left.d_{6}, 500 \mathrm{MHz}\right): \delta=6.30(\mathrm{~s}, 1 \mathrm{H})$, $7.82(2 \mathrm{H}, \mathrm{d}, J=8.75 \mathrm{~Hz}, \mathrm{Ar}), 7.99(2 \mathrm{H}, \mathrm{dd}$, $J=6.50 ; 2.45 \mathrm{~Hz}, \mathrm{Ar}), 8.09$ (1H, dd, $J=6.55 ; 2.40$ $\mathrm{Hz}, \mathrm{Ar}), 8.20\left(2 \mathrm{H}, \mathrm{s}, \mathrm{NH}_{2}\right), 8.23(2 \mathrm{H}, \mathrm{d}, J=8.80$ $\mathrm{Hz}, \mathrm{Ar}), 8.29(1 \mathrm{H}, \mathrm{dd}, J=6.65 ; 2.30 \mathrm{~Hz}, \mathrm{Ar})$ ppm. ${ }^{13} \mathrm{C}$ NMR (DMSO- $\left.d_{6}, 125 \mathrm{MHz}\right): \delta=$ $62.04,115.78,123.79,127.29,128.04,128.98$, 134.66, 145.85, 147.36, 150.93, 153.78, 156.71 ppm. GC-MS: $\mathrm{m} / \mathrm{z}=361\left[\mathrm{M}^{+}\right]$. Anal. calcd. for $\mathrm{C}_{18} \mathrm{H}_{11} \mathrm{~N}_{5} \mathrm{O}_{4}$ : C, 59.84; H, 3.07; N, 19.38. Found: C, 60.02; H, 3.16; N, 19.43 .

\section{3-Amino-1-(4-methoxyphenyl)-5,10-dioxo- 5,10-dihydro-1 $H$-pyrazolo[1,2-b] phthalazine- 2-carbonitrile (4d)}

Yellow crystalline, mp. 264-66 ${ }^{\circ} \mathrm{C}$, FTIR (ATR): $v=3367,3264,3010,2890,2188,1680$, 1655, 1489, 1378, 823, $779 \mathrm{~cm}^{-1} .{ }^{1} \mathrm{H}$ NMR
(DMSO- $\left.d_{6}, 500 \mathrm{MHz}\right): \delta=3.76\left(\mathrm{~s}, 3 \mathrm{H}, \mathrm{OCH}_{3}\right)$, $6.10(\mathrm{~s}, 1 \mathrm{H}, \mathrm{CH}), 6.92(2 \mathrm{H}, \mathrm{d}, J=8.75 \mathrm{~Hz}, \mathrm{Ar})$, $7.39(2 \mathrm{H}, \mathrm{d}, J=8.73 \mathrm{~Hz}, \mathrm{Ar}), 7.89(2 \mathrm{H}, \mathrm{dd}$, $J=5.93 ; 3.30 \mathrm{~Hz}, \mathrm{Ar}), 7.97(1 \mathrm{H}, \mathrm{dd}, J=5.90 ; 3.30$ $\mathrm{Hz}, \mathrm{Ar}), 8.08\left(2 \mathrm{H}, \mathrm{s}, \mathrm{NH}_{2}\right), 8.26(1 \mathrm{H}, \mathrm{dd}, J=5.91$; $3.07 \mathrm{~Hz}$, Ar) ppm. ${ }^{13} \mathrm{C}$ NMR (DMSO- $d_{6}, 125$ $\mathrm{MHz}): \delta=39.35,61.43,113.84,125.13,127.26$, $128.47,128.74,132.58,133.68,134.65,150.57$, 153.59, $156.61 \mathrm{ppm}$. GC-MS: $\mathrm{m} / \mathrm{z}=346\left[\mathrm{M}^{+}\right]$. Anal. calcd. for $\mathrm{C}_{19} \mathrm{H}_{14} \mathrm{~N}_{4} \mathrm{O}_{3}$ : C, 65.89; $\mathrm{H}, 4.07$; N, 16.18. Found: C, 65.93; H, 4.09; N, 16.21.

\section{3-Amino-1-(4-methylphenyl)-5,10-dioxo-5,10- dihydro-1H-pyrazolo[1,2-b] phthalazine-2- carbonitrile (4e)}

Yellow crystalline, mp. 253-55 ${ }^{\circ} \mathrm{C}$, FTIR (ATR): $v=3357,3259,3034,2903,2195,1679$, 1651, 1470, 1378, 823, $734 \mathrm{~cm}^{-1} .{ }^{1} \mathrm{H}$ NMR $\left(\mathrm{DMSO}-d_{6}, 500 \mathrm{MHz}\right): \delta=2.30\left(\mathrm{~s}, 3 \mathrm{H}, \mathrm{CH}_{3}\right)$, 6.09 (s, 1H, CH), 7.17 (2H, d, J=8.02 Hz, Ar), $7.33(2 \mathrm{H}, \mathrm{d}, J=8.06 \mathrm{~Hz}, \mathrm{Ar}), 7.97(2 \mathrm{H}, \mathrm{dd}$, $J=5.90 ; 3.30 \mathrm{~Hz}, \mathrm{Ar}), 8.06\left(2 \mathrm{H}, \mathrm{s}, \mathrm{NH}_{2}\right), 8.09$ $(1 \mathrm{H}, \mathrm{dd}, J=5.80 ; 3.00 \mathrm{~Hz}, \mathrm{Ar}), 8.26(1 \mathrm{H}, \mathrm{dd}$, $J=5.82 ; 3.30 \mathrm{~Hz}, \mathrm{Ar}$ ) ppm. ${ }^{13} \mathrm{C}$ NMR (DMSO- $d_{6}$, $125 \mathrm{MHz}): \delta=20.90,61.48,116.03,126.81$, 127.26, 128.67, 129.04, 133.69, 134.64, 135.38, 150.56, $153.56,156.60 \mathrm{ppm}$. GC-MS: $\mathrm{m} / \mathrm{z}=$ $331\left[\mathrm{M}^{+}\right]$. Anal. calcd. for $\mathrm{C}_{19} \mathrm{H}_{14} \mathrm{~N}_{4} \mathrm{O}_{2}$ : C, 69.08; H, 4.27; N, 16.96. Found: C, 69.13; H, 4.23; N, 16.91 .

\section{3-Amino-1-(2,4-dichlorophenyl)-5,10-dioxo-} 5,10-dihydro- $1 H$-pyrazolo[1,2- $b]$ phthalazine2-carbonitrile (4f)

Pale yellow crystalline, mp. $258-60{ }^{\circ} \mathrm{C}$, FTIR (ATR): $v=3368,3237,3012,2927,2207,1674$, 1657, 1468, 1377, 1277, 840, $720 \mathrm{~cm}^{-1}$. ${ }^{1} \mathrm{H}$ NMR (DMSO- $\left.d_{6}, 500 \mathrm{MHz}\right): 6.45(\mathrm{~s}, 1 \mathrm{H}, \mathrm{CH}), 7.42$ $(1 \mathrm{H}, \mathrm{dd}, J=8.42 ; 2.03 \mathrm{~Hz}, \mathrm{Ar}), 7.67(1 \mathrm{H}, \mathrm{d}$, $J=2.11 \mathrm{~Hz}, \mathrm{Ar}), 7.69(1 \mathrm{H}, \mathrm{d}, J=8.47 \mathrm{~Hz}, \mathrm{Ar})$, 7.99 (2H, dd, J=5.90; $3.20 \mathrm{~Hz}, \mathrm{Ar}), 8.10$ (1H, dd, $J=5.90 ; 3.10 \mathrm{~Hz}, \mathrm{Ar}), 8.17\left(2 \mathrm{H}, \mathrm{s}, \mathrm{NH}_{2}\right), 8.29$ $(1 \mathrm{H}, \mathrm{dd}, J=5.82 ; 3.30 \mathrm{~Hz}, \mathrm{Ar}) \mathrm{ppm} .{ }^{13} \mathrm{C} \mathrm{NMR}$ $\left(\mathrm{DMSO}-d_{6}, 125 \mathrm{MHz}\right): \delta=59.74, \quad 115.56$, 
$126.71,127.35,128.05,128.28,128.84,132.27$, $133.60,133.92,134.76,151.25,153.61,156.68$ ppm. GC-MS: $\mathrm{m} / \mathrm{z}=386\left[\mathrm{M}^{+}\right]$. Anal. calcd. for
$\mathrm{C}_{18} \mathrm{H}_{10} \mathrm{Cl}_{2} \mathrm{~N}_{4} \mathrm{O}_{2}: \mathrm{C}, 56.12 ; \mathrm{H}, 2.62 ; \mathrm{Cl}, 18.41 ; \mathrm{N}$, 14,54. Found: C, 56.15; H, 2.66; Cl, 18.42; N, 14,59 .

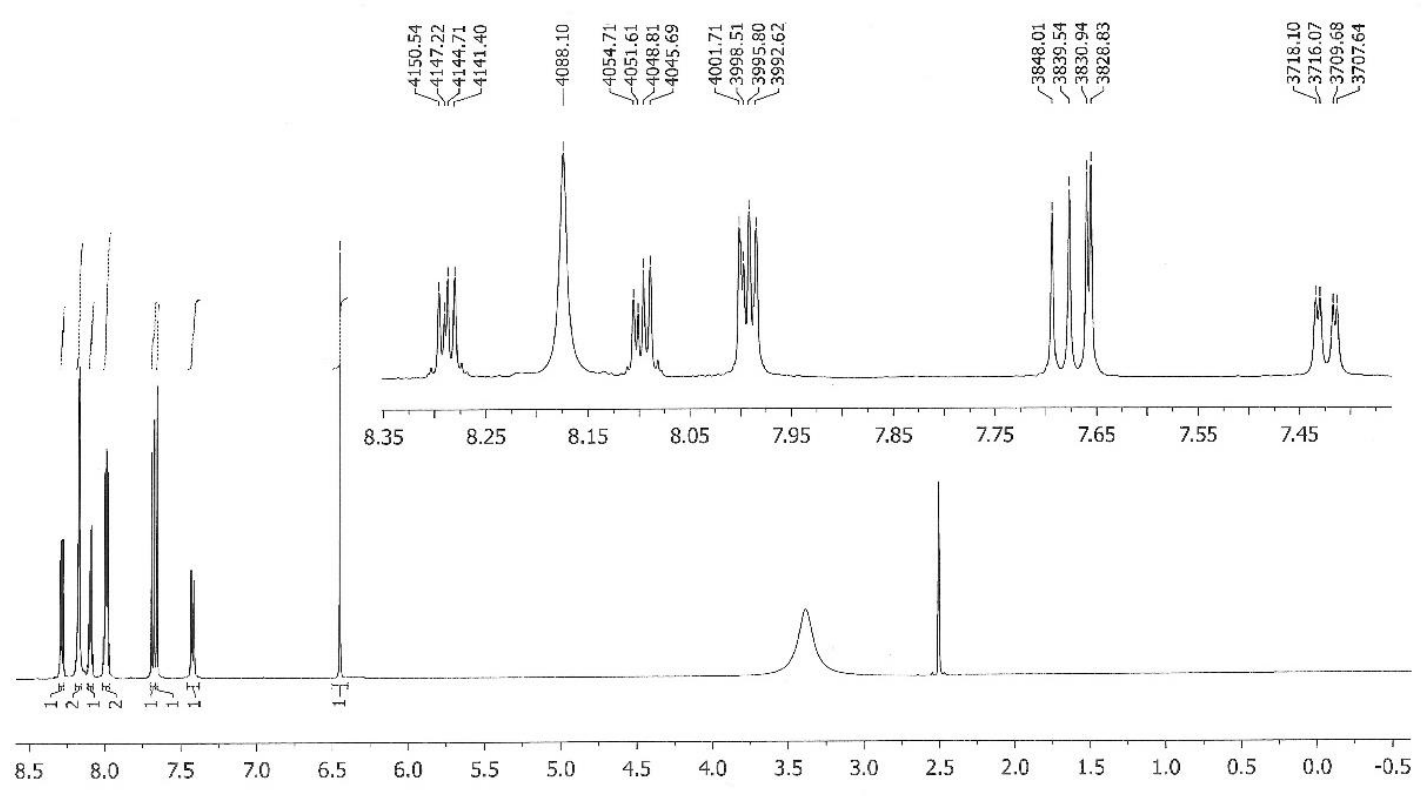

Figure 2. ${ }^{1} \mathrm{H}$ NMR spectrum of $\mathbf{4 f}$ from synthesized compounds
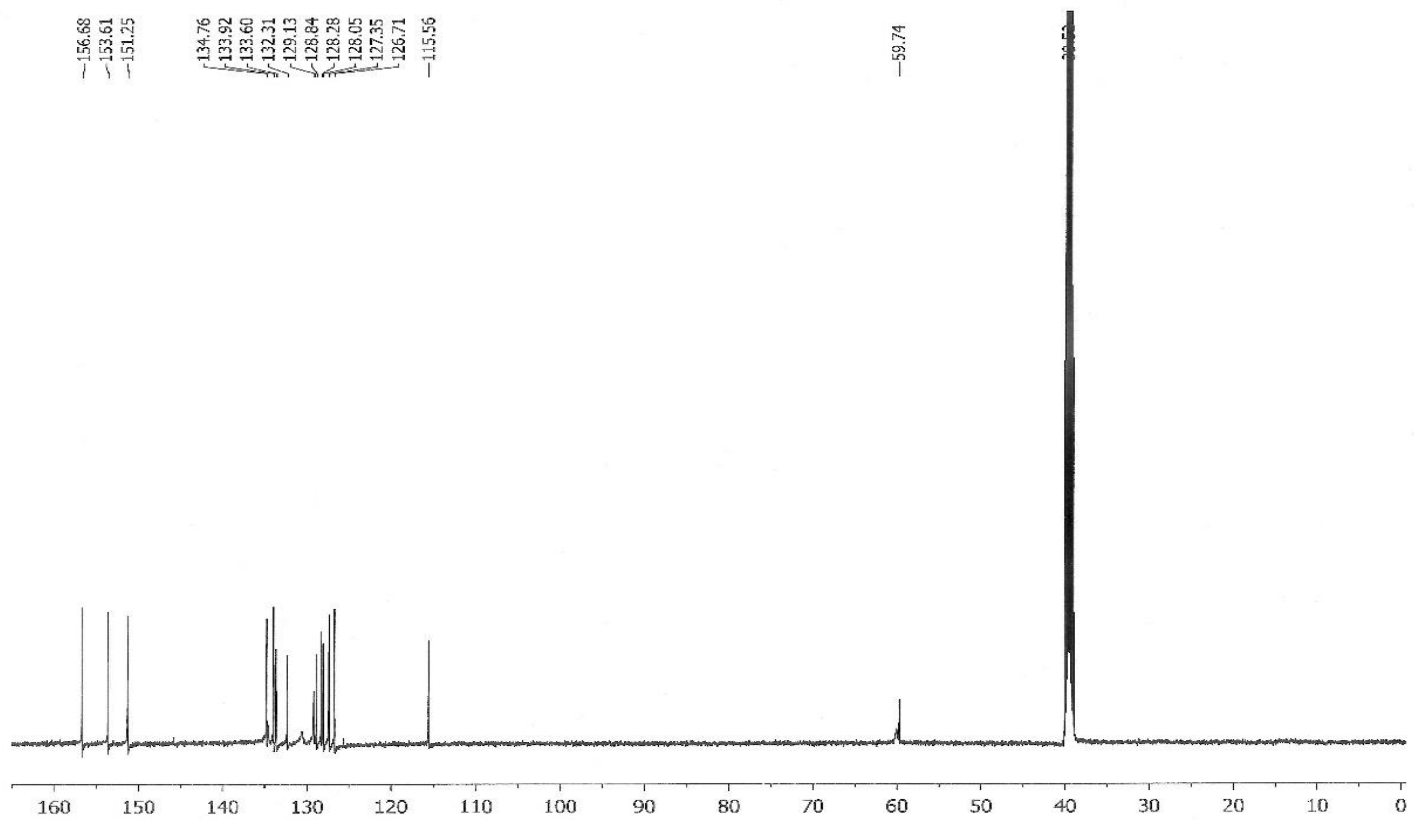

Figure 3. ${ }^{13} \mathrm{C}$ NMR spectrum of $\mathbf{4 f}$ from synthesized compounds 


\section{RESULTS AND DISCUSSION}

Metal triflates have been of great interest as catalysts over the past three decades due to their Lewis acid properties and effective functional group transformations catalyzed by these compounds. It has been well known that lanthanide triflates $\left[\operatorname{Ln}(\mathrm{OTf})_{3}\right]$, and their derivatives are Lewis acids which are stable. It can be used in green chemical methods due to its stability in water. Therefore metal triflates $\mathrm{M}(\mathrm{OTf})_{\mathrm{x}}$ are often used in a variety of organic reactions. Phthalazine derivatives are obtained in the presence of copper(II) triflate as recoverable acid catalysts under moderate reaction conditions by a simple, green and efficient process.

The catalytic effect of $\mathrm{Cu}(\mathrm{OTf})_{2}$ was compared with some metal catalysts which were used widely for this reaction. For this purpose, the one-pot three-component reaction was carried out as a model reaction with phthalhydrazide, benzaldehyde and malononitrile in ethanol (synthesis of 3-amino5,10-dioxo-1-phenyl-5,10-dihydro- $1 \mathrm{H}$ pyrazolo[1,2- $b]$ phthalazine-2-carbonitrile, 4a). It was shown that $\mathrm{Cu}(\mathrm{OTf})_{2}$ was best catalyst among them with lower catalyst amounts. The appropriate amount of $\mathrm{Cu}(\mathrm{OTf})_{2}$ was found to be $10 \mathrm{~mol} \%$. All the results are given in Table 1 .

Table 1. The reactions of phthalhydrazide, 1,3-cyclohexadione and benzaldehyde (4a); effect of catalysts. ${ }^{a}$

\begin{tabular}{cccccc}
\hline Entry & Catalyst & Used reactions & $\begin{array}{c}\text { Amount of catalyst } \\
(\mathbf{m o l} \%)\end{array}$ & Time (h) $^{(\mathbf{m})}$ & Yield (\%) $^{\mathbf{b}}$ \\
\hline 1 & $\mathrm{None}$ & $4 \mathrm{a}$ & --- & 24 & 38 \\
2 & $\mathrm{AlCl}_{3}$ & $4 \mathrm{a}$ & 100 & 24 & 47 \\
3 & $\mathrm{ZnCl}_{2}$ & $4 \mathrm{a}$ & 100 & 24 & 45 \\
4 & $\mathrm{FeCl}_{3}$ & $4 \mathrm{a}$ & 100 & 24 & 48 \\
5 & $\mathrm{Cu}(\mathrm{OTf})_{2}$ & $4 \mathrm{a}$ & 10 & 2 & 82 \\
6 & $\mathrm{Cu}(\mathrm{OTf})_{2}$ & $4 \mathrm{a}$ & 15 & 2 & 89 \\
7 & $\mathrm{Cu}(\mathrm{OTf})_{2}$ & $4 \mathrm{a}$ & 20 & 2 & 89 \\
8 & $\mathrm{Cu}(\mathrm{OTf})_{2}$ & $4 \mathrm{a}$ & & & 87 \\
\hline
\end{tabular}

${ }^{a}$ All reactions were carried out under reflux in ethanol.

${ }^{\mathrm{b}}$ Isolated yields.

A mechanism for the activity of $\mathrm{Cu}(\mathrm{OTf})_{2}$ in the synthesis of $1 H$-pyrazolo[1,2- $b$ ] phthalazine5,10-dione derivatives could be postulated as shown in Figure 4 (Reddy and Jeong, 2013; Eswararao et al., 2017).

The reaction possible take shape by Knoevenagel condensation between aldehyde (2) and malononitrile (3) on the acidic activity of $\mathrm{Cu}(\mathrm{OTf})_{2}$ to form 5 . Then Michael type addition to 2,3-dihydrophthalazine-1,4-dione (1), followed by ring closure and tautomerization to form $4 \mathbf{a}-\mathbf{f}$.
Under optimized conditions compounds $4 \mathrm{a}-\mathrm{f}$ were synthesized with different aldehydes with high yields and short reaction time as shown in the Table 2. The structures of known compounds (4a-f) were in accordance with their spectral data (Ghorbani-Vaghei et al., 2014; Abdesheikhi and Karimi-Jaberi, 2015; Ghorbani-Vaghei et al., 2016; Kerayati et al., 2016; Mohamadpour et al., 2016; Wang et al., 2016; Arora and Rajput, 2017; Eswararao et al., 2017; Tayade and Dalal, 2017; Sabour et al., 2018; Shaikh et al., 2018). 


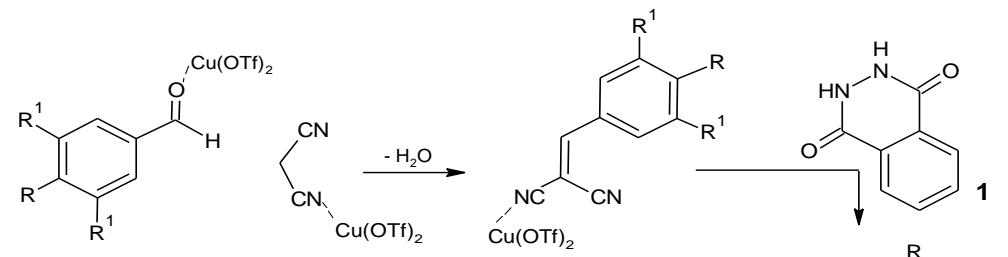

2

3

5

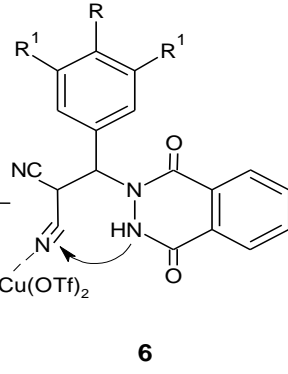

(1)

$\mathrm{Cu}(\mathrm{QTf})_{2}$

$-\mathrm{Cu}(\mathrm{OTf})_{2}$
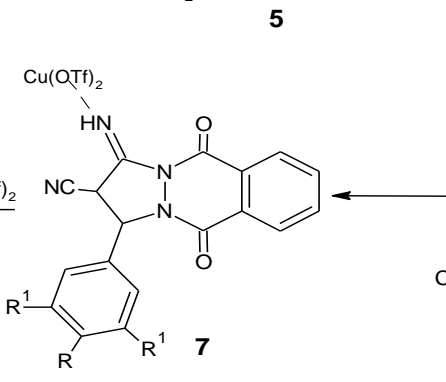

$\mathrm{R}=\mathrm{H}, \mathrm{Br}, \mathrm{NO}_{2}, \mathrm{OCH}_{3}, \mathrm{CH}_{3}$

$\mathrm{R}^{1}=\mathrm{H}, \mathrm{Cl}$

Figure 4. A schematic mechanism for the activity of $\mathrm{Cu}(\mathrm{OTf})_{2}$ in the synthesis of $1 H$-pyrazolo[1,2$b$ ]phthalazine-5,10-dione derivatives.

Table 2. Preparation of $1 H$-pyrazolo[1,2- $b]$ phthalazine-5,10-dione derivatives ${ }^{\mathrm{a}}$.

\begin{tabular}{llllll}
\hline Entry & $\mathbf{R}$ & $\mathbf{R}^{\mathbf{1}}$ & Product & Time & Yield (\%) $^{\mathbf{b}}$ \\
\hline $\mathbf{1}$ & $\mathrm{H}$ & $\mathrm{H}$ & $4 \mathrm{a}$ & $2 \mathrm{~h}$ & 89 \\
$\mathbf{2}$ & $\mathrm{Br}$ & $\mathrm{H}$ & $4 \mathrm{~b}$ & $2 \mathrm{~h}$ & 91 \\
$\mathbf{3}$ & $\mathrm{NO}_{2}$ & $\mathrm{H}$ & $4 \mathrm{c}$ & $3 \mathrm{~h}$ & 93 \\
$\mathbf{4}$ & $\mathrm{CH}_{3} \mathrm{O}$ & $\mathrm{H}$ & $4 \mathrm{~d}$ & $2 \mathrm{~h}$ & 89 \\
$\mathbf{5}$ & $\mathrm{H}$ & $\mathrm{Cl}$ & $4 \mathrm{e}$ & $2 \mathrm{~h}$ & 87 \\
$\mathbf{6}$ & $\mathrm{CH}_{3}$ & $\mathrm{H}$ & $4 \mathrm{f}$ & $2 \mathrm{~h}$ & 86 \\
\hline
\end{tabular}

${ }^{\mathrm{a}}$ All reactions were carried out in ethanol at $80^{\circ} \mathrm{C}$ in the presence of $\mathrm{Cu}(\mathrm{OTf})_{2}(10 \mathrm{~mol} \%)$.

${ }^{\mathrm{b}}$ Isolated yields

To investigate the catalyst activity, the the completion of each reaction, $\mathrm{Cu}(\mathrm{OTf})_{2}$ was catalyst was reused six times in the one-pot washed with hot water and filtered. The multi-component condensation of recovered $\mathrm{Cu}(\mathrm{OTf})_{2}$ was dried and reused. As phthalhydrazide, benzaldehyde and shown in Figure 5, very low losses were malononitrile at $80{ }^{0} \mathrm{C}$. In this procedure, after observed in the catalytic activity of $\mathrm{Cu}(\mathrm{OTf})_{2}$.

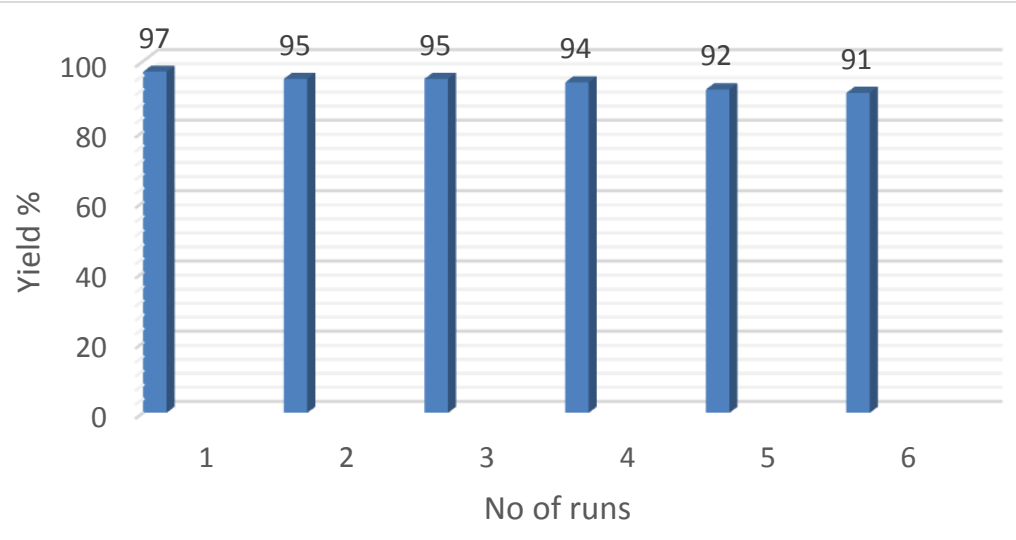

Figure 5. Reusability of the catalyst in the reaction. 


\section{CONCLUSION}

In the study, it has been found that the synthesis of bioactive phthalazine derivatives with metal triflate takes place in excellent yields and short times, and metal triflates have an environmentally beneficial, economically applicable and reusable catalytic capability. Using this method offers many advantages under solvent-free conditions, which are considered to be relatively eco-friendly, such as very good yield, low catalyst amount, less reaction time, easier handling and multi-component reaction. Therefore, this article is thought to lead to future studies.

\section{ACKNOWLEDGMENTS}

This work was supported by Research Fund of the Yildiz Technical University. Project Number: 2012-01-02-GEP01.

\section{REFERENCES}

Abdesheikhi M, Karimi-Jaberi Z, 2015. Fourcomponent synthesis of 3-amino-1-aryl5,10-dioxo- $1 H$-pyrazolo[1,2- $b]$

phthalazine-2-carbonitrile derivatives promoted by potassium carbonate. Chemical Research, 39: 482-483.

Arora P, Rajput JK, 2017. Amelioration of $\mathrm{H}_{4}\left[\mathrm{~W}_{12} \mathrm{SiO}_{40}\right]$ by nanomagnetic heterogenization: For the synthesis of $1 \mathrm{H}-$ pyrazolo[1,2- $b]$ phthalazinedione derivatives. Apllied Organometallic Chemistry, 32: 1-18.

Bashti A, Kiasat AR, Mokhtari B, 2015. Synthesis and characterization of dicationic 4,4'-bipyridinium dichloride ordered mesoporous silica nanocomposite and its application in the preparation of $1 H$-pyrazolo[1,2- $b]$ phthalazine-5,10-dione derivatives. Royal Society of Chemistry Advances, 5: 25816-25823.
Dabholkar V, Badhe K, Kurade S, 2017. Onepot four component synthesis of $1 \mathrm{H}$ pyrazolo $\quad[1,2-b]$ phthalazine-5,10-dione derivatives using calcined $\mathrm{Mg}-\mathrm{Fe}$ hydrotalcite catalyst. Internationl Journal of Current Advanced Research, 6:84168420 .

Dabholkar V, Kurade S, Badhe K, 2018. Synthesis of $1 H$-pyrazolo[1,2$b$ ]phthalazine-5,10-dione derivatives using $\mathrm{NiFe}_{2} \mathrm{O}_{4}$ nanoparticle as a heterogeneous catalyst. Der Pharma Chemica, 10: 135141.

Eswararao S, Venkataramireddy V, Sreenivasareddy M, Kumar P, 2017. Water mediated one-pot synthesis of $1 \mathrm{H}$ pyrazolo[1,2- $b]$ phthalazine-5,10-diones and $\quad 2 H$-indazolo[2,1-b]phthalazine1,6,11(13H)-triones, Heterocyclic Letters, 7(3): 895-903.

Ghahremanzadeh R, Shakibaei GI, Bazgir A, 2008. An efficient one-pot synthesis of $1 H$-pyrazolo[1,2- $b]$ phthalazine-5,10-dione derivatives. Synlett, 8:1129-1132.

Ghomi JS, Alavi HS, Ziarati A, Teymuri R, Saberi MR, 2014. A highly flexible green synthesis of $1 H$-pyrazolo[1,2$b$ ]phthalazine-5,10-dione derivatives with $\mathrm{CuI}$ nanoparticles as catalyst under solvent-free conditions. Chinese Chemical Letters, 25:401-405.

Ghorbani-Vaghei R, Noori S, ToghraeiSemiromi Z, Salimi Z, 2014. One-pot synthesis of $1 H$-pyrazolo[1,2$b$ ]phthalazine-5,10-dione derivatives under solventfree conditions. Royal Chemical Society Advances 4: 47925-47928. 
Ghorbani-Vaghei R, Mahmoodi J, Maghbooli Y, 2016. Preparation and characterization of nanomagnetic piperidinium benzene- 1,3disulfonate ionic liquid as a novel, green and heterogeneous catalyst and its use in the synthesis of $1 H$-pyrazolo[1,2b]phthalazine- 5,10- diones and $1 H_{-}$ pyrazolo[1,2- $a$ ]pyridazine- 5,8- diones under solvent- free conditions, Apllied Organometallic Chemistry, 31: 1-10.

Hosseininasab N, Davoodnia A, Rostami-Charati F, Khojastehnezhad A, 2017. Preparation, characterization, and first catalytic application of a novel phosphotungstic acid-containing ionic liquid immobilized on $\mathrm{CuFe}_{2} \mathrm{O}_{4} @ \mathrm{SiO}_{2}$ magnetic nanoparticles in the synthesis of $1 H$-pyrazolo[1,2b]phthalazine-5,10-diones. Russian Journal of General Chemistry, 87(10): 2436-2443.

Kefayati H, Amlashi SH, Kazemi-Rad R, Delafrooz A. 2014. Electrocatalytic multicomponent assembling of phthalhydrazide, aldehydes and malononitrile: An efficient approach to $1 H$-pyrazolo[1,2- $b]$ phthalazine-5,10diones. Comptes Rendus Chimie. 17: 894898.

Kefayati H, Delafrooz A, Homayoon S, 2016. Ultrasound-Assisted synthesis of pyrazolo[1,2- $b]$ phthalazines and dihydrospiro[indoline-3,1'-pyrazolo[1,2$b$ ]phthalazines] using TBAF as an efficient phase-transfer catalyst. Russian Journal of General Chemistry, 86(7): 1735-1740.

Kiasat AR and Davarpanah J, 2013. $\mathrm{Fe}_{3} \mathrm{O}_{4} @$ silica sulfuric acid nanoparticles: An efficient reusable nanomagnetic catalyst as potent solid acid for one-pot solvent-free synthesis of indazolo[2,1-b] phthalazine-triones and pyrazolo[1,2$b$ ]phthalazine-diones. Journal of Molecular Catalysis A: Chemical, 373: 46-54.
Kobayashi S, Sugiura M, Kitagawa H, Lam WWL, 2002. Rare-earth metal triflates in organic synthesis, Chemical Reviews, 102(6): 2227-2302.

Lamera E, Bouacida S, Merazig H, Chibani A, Le Borgne M, Bouaziz Z, Bouraiou A, 2017. DMAP as a new efficient catalyst for the one-pot synthesis of condensed phthalazines. Zeitschrift für Naturforschung B, 72:361-368.

Lv PC, Sun J, Luo Y, Yang Y, Zhu HL, 2010. Design, synthesis, and structure-activity relationships of pyrazole derivatives as potential FabH inhibitors. Bioorganic \& Medicinal Chemistry, 20: 4657-4660.

Mohamadpour F, Maghsoodlou MT, Heydari R, 2016. Mojtaba Lashkari2Saccharin: a green, economical and efficient catalyst for the one- pot, multi- component synthesis of 3,4-dihydropyrimidin-2- $(1 H)^{-}$one derivatives and $1 H^{-}$pyrazolo[1,2- $\left.b\right]$ phthalazine- $5,10^{-} \mathrm{d}$ ione derivatives and substituted

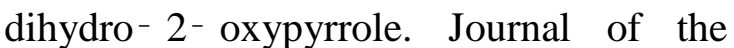
Iranian Chemical Society, 13:1549-1560.

Mohamadpour F, Lashkari M, Heydari R, Hazeri N, 2018. Four-component clean process for the eco-friendly synthesis of $1 \mathrm{H}$ pyrazolo $\quad[1,2-b]$ phthalazine-5,10-dione derivatives using $\mathrm{Zn}(\mathrm{OAc})_{2} \cdot 2 \mathrm{H}_{2} \mathrm{O}$ as an efficient catalyst under solvent-free conditions. Indian Journal of Chemistry, 57B:843-851.

Nabid MR, Rezaei SJT, Ghahremanzadeh R, Bazgir A, 2010. Ultrasound-assisted onepot, three-component synthesis of $1 \mathrm{H}$ pyrazolo[1,2- $b]$ phthalazine-5,10-diones. Ultrasonics Sonochemistry, 17: 159-161. 
Piltan M, 2017. Preparation of $1 H$-pyrazolo[1,2$b]$ phthalazine-5,10-diones using $\mathrm{ZrO}_{2}$ nanoparticles as a catalyst under solventfree conditions. Heterocyclic Communications, 23:401-403.

Raghuvanshi DS and Singh KN, 2011. A highly efficient green synthesis of $1 H$ pyrazolo[1,2-b]phthalazine-5,10-dione derivatives and their photophysical studies. Tetrahedron Letters, 52: 5702-5705.

Reddy MV and , Jeong YT, 2013. $\mathrm{InCl}_{3^{-}}$ catalyzed green synthesis of $1 \mathrm{H}$ pyrazolo[1,2- $b]$ phthalazine-5,10-diones under solvent-free conditions. Tetrahedron Letters, 2013, 54(27): 3546-3549.

Reddy MV, Kumar PCR, Reddy GCS, Reddy CS, 2014. Silica gel-supported tungstic acid (STA): A new, highly efficient and recyclable catalyst for the synthesis of $1 \mathrm{H}$ pyrazolo[1,2-b]phthalazine-5,10-dione carbonitriles and carboxylates under neat conditions. Comptes Rendus Chimie, $17: 1250-1256$

Roy HN, Rana M, Al Munsur AZ, Lee K, Sarker AK, 2016. An efficient and convenient synthesis of $1 H$-pyrazolo[1,2$b$ ]phthalazine-5,10-dione derivatives mediated by L-proline. Synthethic Communications, 46:1370-1376.

Sabour $\quad \mathrm{FH}, \quad$ Nasr- Esfahan

M, Mohammadpoor- Baltork

Tangestaninejad S, Moghadam M, Mirkhani V, 2018. A convenient approach for the synthesis of various derivatives of pyrazolo[1,2- $b]$ phthalazinediones in the presence of an efficient supported basic ionic liquid at ambient temperature and solvent- free media. Journal of the Iranian Chemical Society, 15:671-683.
Sangani CB, Makwana JA, Duan YT, Thumar NJ, Zhao MY, Patel YS, Zhu HL, 2016. Synthesis of $1 H$-pyrazolo[1,2b]phthalazine-5,10-dione derivatives: Assessment of their antimicrobial, antituberculosis and antioxidant activity. Research on Chemical Intermediates, 42:2101-2117.

Shah NM, Patel MP, Patel RG, 2012. An efficient and facile synthesis of $1 \mathrm{H}$ pyrazolo[1,2- $b]$ phthalazine- 5,10 - dione derivatives of biological interest. Heterocyclic Chemistry, 49:1310-1316.

Shaikh MA, Farooqui M, Abed S, 2018. $\left[\mathrm{Bu}_{3} \mathrm{NH}\right]\left[\mathrm{HSO}_{4}\right]$ catalyzed: an ecoefficient synthesis of $1 H$-pyrazolo[1,2b]phthalazine-5,10-diones and $2 \mathrm{H}$ indazolo[2,1- $b]$ phthalazine-triones under solvent-free conditions. Research on Chemical Intermediates, 44: 5483-5500.

Shaterian HR, Mohammadnia M, 2012. Mild basic ionic liquids catalyzed new fourcomponent synthesis of $1 H$-pyrazolo[1,2$b$ ]phthalazine-5,10-diones, Journal of Molecular Liquids, 173:55-61.

Song SH, Zhong J, He YH, Guan Z, 2012. Onepot four-component synthesis of $1 \mathrm{H}$ pyrazolo[1,2-b]phthalazine-5,10-dione derivatives. Tetrahedron Letters, 53:70757077 .

Tayade YA, Dalal DS, 2017. $\beta$-Cyclodextrin as a supramolecular catalyst for the synthesis of $1 H$-pyrazolo[1,2- $b]$ phthalazine-5,10-dione derivatives in water. Catalysis Letters, 147:1411-1421.

Teimouri MB, 2006. One-pot three-component reaction of isocyanides, dialkyl acetylenedicarboxylates and phthalhydrazide: synthesis of highly functionalized $\quad 1 H$-pyrazolo[1,2b]phthalazine-5,10-diones. Tetrahedron, 62:10849-10853. 
Torkiana L, Dabiri M, Salehi P, Bararjaniana M, 2011. An efficient one-pot, fourcomponent synthesis of $\{[(1 H-1,2,3-$ triazol-4-yl)methoxy]phenyl $\}-1 H$ pyrazolo[1,2-b]phthalazine-5,10-dione derivatives. Helvetica Chimica Acta, 94: 1416-1425.

Vafaee A, Davoodnia A, Pordel M, Bozorgmehr MR, 2015. An efficient and high-yielding one-pot synthesis of $1 H$-pyrazolo[1,2$b]$ phthalazine-5,10-diones catalyzed by sodium hydrogen carbonate under solventfree conditions. Oriental Journal of Chemistry, 31:2153-2158.

Vaghei RG, Noori S, Semiromi ZT, Salimi Z, 2014. One-pot synthesis of $1 H$ pyrazolo[1,2- $b]$ phthalazine-5,10-dione derivatives under solvent-free conditions. Royal Society of Chemistry Advances, 4:47925-47928.

Vera-DiVaio MAF, Freitas ACC, Castro HC, de Albuquerque S, Cabral LM, Rodrigues CR, Albuquerque MG, Martins RCA, Henriques MGMO, Dias LRS, 2009. Synthesis, antichagasic in vitro evaluation, cytotoxicity assays, molecular modeling and SAR/QSAR studies of a 2-phenyl-3(1-phenyl-1H-pyrazol-4-yl)-acrylic acid benzylidene-carbohydrazide series. Bioorganic \& Medicinal Chemistry, 17: 295-302.

Wang W, Cong-Hao L, Yi Y, Xiao-Jun L, HongYun G, 2016. An improved procedure for the three-component synthesis of $1 \mathrm{H}$ pyrazolo[1,2- $b]$ phthalazine-5,10-dione derivatives using basic ionic liquid. Journal of Chemical Research, 40: 354357.
Wei F, Zhao BX, Huang B, Zhang L, Sun CH, Dong WL, Shin DS, Miao JY, 2006. Design, synthesis, and preliminary biological evaluation of novel ethyl 1-(2'hydroxy-3'-aroxypropyl)-3-aryl-1Hpyrazole-5-carboxylate. Bioorganic \& Medicinal Chemistry Letters, 16: 63426347.

Wender PA, Handy ST, Wright DL, 1997. Towards the ideal synthesis. Chemistry and Industry. 19: 765-768.

Xia Y, Dong ZW, Zhao BX, Ge X, Meng N, Shin DS, Miao JY, 2007. Synthesis and structure-activity relationships of novel 1arylmethyl-3-aryl-1 $H$-pyrazole-5-

carbohydrazide derivatives as potential agents against A549 lung cancer cells. Bioorganic \& Medicinal Chemistry, 15: 6893-6899.

Zhang S, Zhao Y, Liu Y, Chen D, Lan W, Zhao Q, 2010. Synthesis and antitumor activities of novel 1,4-disubstituted phthalazine derivatives. European Journal of Medicinal Chemistry, 45(8): 3504-3510. 